\title{
SCRUTINY OF BROADCASTING PROTOCOLS IN VANET
}

\author{
Rohini Nere and Prof Uma Nagaraj \\ Department of Computer Engineering, MIT Academy of Engineering, Alandi, Pune
}

\begin{abstract}
Definitive broadcasting in vehicular ad hoc networks is the key to success for various services and applications on an intelligent transportation system. Many trusted broadcasting protocols have been proposed but none of them has been evaluated in realistic scenario. In this paper, we discuss these reliable broadcasting protocols on VANET. Basic mechanism in broadcasting, and also we are providing collective research of Broadcasting protocols in vanet in that some real time protocols with their pros cons we have studied.
\end{abstract}

\section{KEYWORDS}

Vanet, broadcasting protocols , flooding, DSR, UMB, AODV, MDDV.

\section{INTRODUCTION}

There are several broadcasting protocols proposed on Vehicular Adhoc Network. But as of now none of them has been successfully evaluated. Hence, authors of this paper have done analytic research of the broadcasting protocols for vehicular Adhoc Network.

\section{NEED OF BROADCASTING IN VANET}

A conventional approach for broadcasting is simple flooding. As soon as node receive broadcast message receiving node rebroadcasts the message immediately. This approach can provide very high rate of data distribution. It is also simple as it does not require neighbour's information. However, it does not execute well in dense and sparse areas. Particularly, in condensed area such as traffic jam during rush hours, the simple flooding is responsible for high collision, leading to low reliability with a lot of dispensable broadcast messages. This problem is also been applicable for route request (RREQ) mechanism of AODV on the Vehicular AdHOc Network environment. In sporadic area such as highways during night time, vehicles move fast and possibly have no neighbour in their transmission range. The effortless flooding in such disconnected network is useless as there is no neighbour being able to receive and convey the message VANET safety mechanism depend on interchanging the safety information among vehicles i.e. car to car (C2C communication) or between Vehicle to infrastructure i.e. car to infrastructure.(C2I Communication) using the control channel, as you can see in figure 1. 


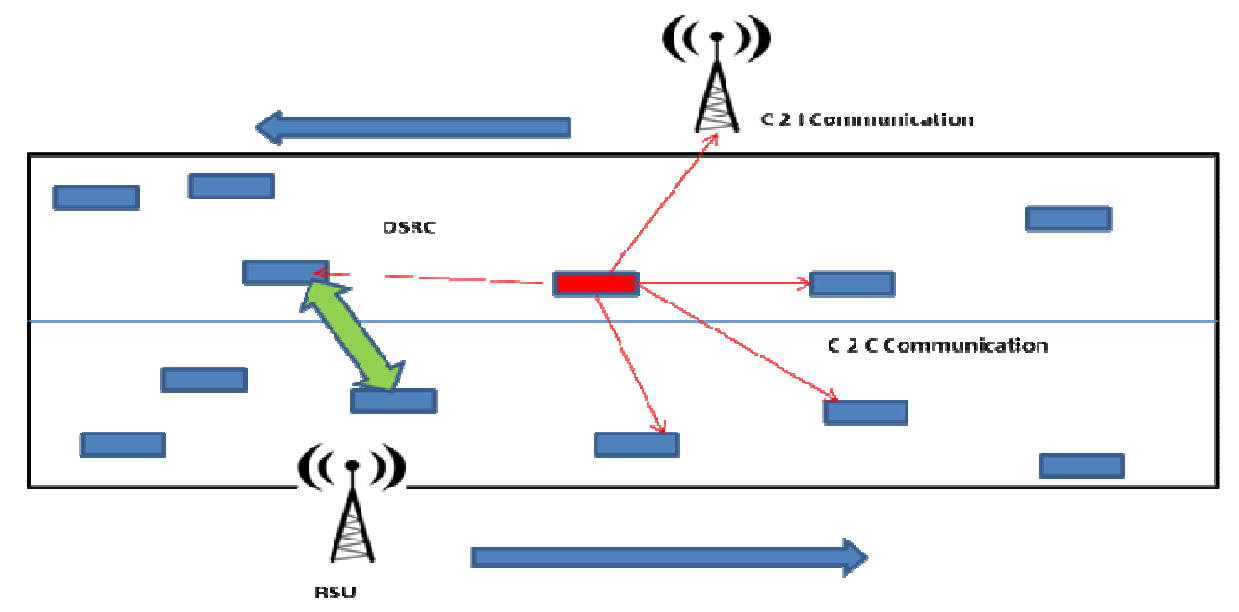

Figure 1: Basic idea of VANET.

VANET as comfort communication can be made by two means: Periodic Safety Message (in this paper we refer them as Beacon) and Event Driven Message (refereed as Emergency Message here), both messages share only one control channel. The Beacon messages are messages about status of sender vehicle. Status information includes position, speed, heading towards, etc., about sender. Beacons provide resent or latest information of the sender vehicle to the all present vehicles in the network which will help them to know the position of the current network and anticipate the movement of vehicles. Beacons are sent antagonistically to neighbouring vehicles 10 messages each second. Emergency Messages are messages sent by a vehicle who defect a potential dangerous situation on the road, this information should be dispersed to alarm or worn other vehicles about a feasible danger that could affect the incoming vehicles. VANET is a high mobile or volatile network where the nodes are keep changing their position and they are moving in speeds, which means that this vehicles may be get influence, even if these vehicles are very far from the danger, they will reach near to danger very soon, in this case fraction of seconds will be very important to avoid the danger [6, and 7].

In VANET Emergency messages are delivered in broadcasting way. Purpose behind this is all the vehicle within the communication range of the sender should receive the message. Message barely reaches a $1000 \mathrm{~m}$ (which is in fact the range of DSRC communication) and is the coverage area of sender and it is not enough as due to fading effects and attenuation. Critical information should receive by vehicles which are out of senders range. By using this information they can avoid the danger. In short distances the prospect of message reception i.e. percentage of message reception can reach $99 \%$ and as we move forward it decreases up to $20 \%$ at half of the communication range (Moreno, 2004). Therefore, there is requirement of a method to rise the emergency message reception with great reliability and availability. Due to the high movement of vehicles, the dispersal of nodes with in the network changes swiftly and unpredictably that wireless links modify and clear up commonly and randomly. Therefore, broadcasting of messages in VANETs plays a pivotal rule in almost every application and requires novel solutions that are different from any other form of Ad-Hoc networks. Broadcasting of the messages in VANETs is an open investigation challenge and needs some efforts to reach an optimum solution.

The requirements for broadcasting are great accuracy, dependability and also great distribution speed by means of undersized latency not only in single-hop but also in multi-hop communication. 
Problems associated with regular broadcasting algorithms are the high possibility of collision in the broadcasted messages and the absence of feedback and the hidden node problem. In our paper we are proposing a new intelligent broadcasting technique which is used in emergency message in Vehicular AdHoc Network and is aimed in rising of the acknowledgement of important message.

In section II and section III we are simply collecting all possible information about research done in broadcasting protocols. In that section II is dedicated to schemes of existing broadcasting protocols. And section III is describing some real time examples of broadcasting protocols with their pros and cons.

\section{Basic Broadcasting Schemes Comparison}

A variety of broadcasting schemes exist such as simple flooding, probability based approaches, area based approaches etc., In this section we will concisely discuss all the broadcast schemes and their pros and cons. Flooding is a simple broadcast technique for communication. Vehicles send information to other vehicle and this process continues until all vehicles get same information. It works fine in sparse network but in dense network it produces collision, contention and redundant information. Flooding is pretty simple to practise and also flooding could be expensive as far as network performance is concerned, and one of the main problems that rise in flooding is "Broadcast Storm Problem". Figure 2 is showing difficulty with flooding vehicle in yellow colour first send message. Which is again come back after some interval from different user's repetition of message take place which results into wastage of bandwidth.

Red line indicates that message is repetitively sanded back

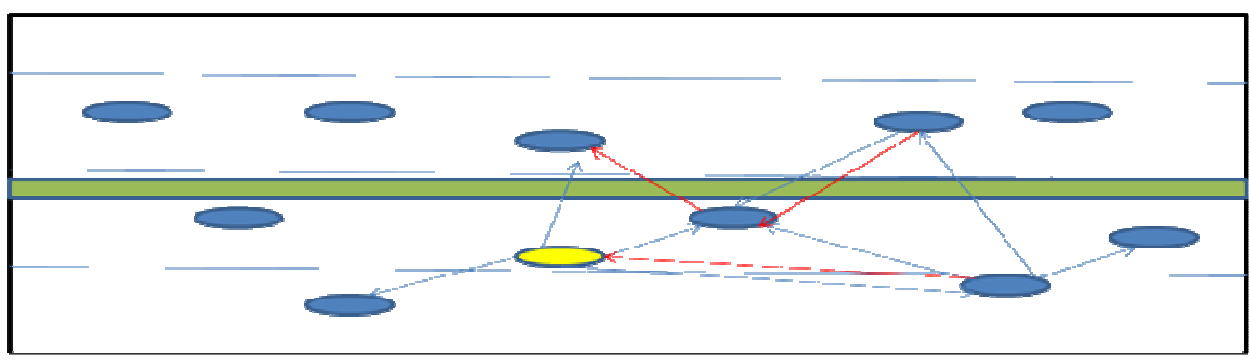

Figure 2. Broadcast storm problem

Probabilistic scheme reduces the collision, contention and redundant messages in dense network as it broadcast the messages with some fixed probability. However in the scattered network, all of the vehicles are unable to receive the same packets with small probability. When the possibility is increased then it mechanisms similar to flooding. Therefore, it performs wisely in condensed network in comparison to scattered network. Counter based technique is used to analyse the redundant messages. We use counter to record the dispensable message. Whenever the redundant i.e. dispensable message is received, we increment the counter by one. We distinguish the counter with certain threshold value if it is less than it we forward the packet otherwise the packet is discarded. The Scheme based on Distance, it evaluates the space between the vehicles and its neighbours. Then it compares the distance with threshold. If the distance is greater than threshold it forward the packet otherwise it ignore the message. The Scheme based on Location, it firstly computes the coverage area by making use of sender location. If the coverage area is shorter than a threshold value, then the vehicle will overlook the packet. Or else that packet would be broadcasted. Neighbour knowledge method is a technique which keeps the table which comprises of the data for vehicles which are nearby. A vehicle uses this information to decide whether to 
forward the message onward or deny it. All vehicles share hello packets with their neighbours to get current information. That information is being stored in their table for further usage. This method completely depends on the exchange of hello packet. If the intermission is little then Contention and collision can happen, whereas the huge intermission worsens the network performance because of motion.

Correlation of various broadcasting protocols is being presented below in table. We have done analysis on the protocols on the basis of different criterions viz. are, collision, competition, congestion, performance, accuracy, reliability. And not any of the previously existing schemes is perfect for the entire situations .In sparse or scattered network, plain flooding could behave properly whereas the probabilistic can work well in condensed network.

Table 1. Comparison between protocols.

\begin{tabular}{|l|l|l|l|l|l|l|l|}
\hline \multirow{2}{*}{ No } & \begin{tabular}{l} 
Characteristics \\
\cline { 2 - 7 } \\
Protocols
\end{tabular} & Reliability & Performance & Congestion & Rebroadcast & Collision & Contention \\
\hline 1 & Simple Flooding & Quite high & Limited & Very high & Spare & Very high & high \\
\hline 2 & $\begin{array}{l}\text { Probabilistic } \\
\text { Scheme }\end{array}$ & Limited & Limited & Little & Controlled & Limited & low \\
\hline 3 & $\begin{array}{l}\text { Counter Based } \\
\text { Scheme }\end{array}$ & Limited & Limited & Little & Controlled & Limited & low \\
\hline 4 & $\begin{array}{l}\text { Distance Based } \\
\text { Approach }\end{array}$ & Limited & Limited & Little & Controlled & little & Limited \\
\hline 5 & $\begin{array}{l}\text { Location Based } \\
\text { Approach }\end{array}$ & high & high & Little & Efficient & little & Limited \\
\hline 6 & $\begin{array}{l}\text { Neighbour } \\
\text { Knowledge } \\
\text { Method }\end{array}$ & Limited & Limited & Limited & controlled & $\begin{array}{l}\text { Period } \\
\text { dependent } \\
\text { (Limited) }\end{array}$ & $\begin{array}{l}\text { Period } \\
\text { dependent } \\
\text { (Limited }\end{array}$ \\
\hline 7 & $\begin{array}{l}\text { Tree Based } \\
\text { Broadcast }\end{array}$ & high & Little & high & Very low & Efficient & Very low \\
\hline 8 & UMB & high & High & Limited & controlled & little & Limited \\
\hline 9 & MDDV & high & High & Low & controlled & little & low \\
\hline 10 & $\begin{array}{l}\text { Relevance-based } \\
\text { approach }\end{array}$ & Limited & High & Low & Efficient & little & low \\
\hline 11 & MHVB & high & High & Very low & Efficient & high & low \\
\hline 12 & $\begin{array}{l}\text { Adaptive } \\
\text { broadcast } \\
\text { protocol }\end{array}$ & high & High & Limited & Inefficient & high & high \\
\hline
\end{tabular}

\subsection{Probe of Broadcasting Protocols in Vanet}

Basic mechanisms in which consist of three main modules. There are favoured node selection algorithm, beaconing and waiting timeout calculation. The preferred node selection (node picking) algorithm which can make the difference to efficiency and overhead of the protocol. If the protocol picks the exact vehicle, some of the vehicles in the similar road sector would be able to accept the broadcasted message in every single phase of rebroadcasting. The waiting timeout calculation is the chief mechanism to circumvent broadcasting collision for reliable (consistent) broadcasting protocol, nevertheless waiting timeout could raise a lot of retard. We also found that the waiting timeout of some protocols is the reason of broadcast storm problem. The last module is beaconing which is the solution to redeem neighbour nodes' information. Even though it is beneficial, it is able to spend the inadequate wireless network resource, which origins problem of broadcasting storm which then diminish consistency and efficiency of protocols. Then we compare the reliable broadcast protocols on the basis of reliability (dependability), speed and overhead of data scattering by imitation. 
In the reference no [8], a street-based broadcast scheme is proposed by authors in that scheme vehicle employs neighbours' information by exchanging hello messages among vehicles, A warning message is broadcasted to all neighbours when any probable danger is detected. As a forwarder the farthest vehicle is selected. This selection will be depending on the information gained from the hello message, if the forwarder which is preselected, receives the message, it will broadcast it again.

In a case of high mobile highly volatile network like VANET Depending on just one forwarder is not good option always. Moreover, we are not dependant on beacon signals to intensification of the information. They anticipated using hello message, which creates a chance to raise the channel load. The waiting time for long the receiver delays previous to rebroadcasting of the message acknowledged by the sender is the contention period. And these schemes are anticipated by many of the researchers [10], [12], [9], [13], [11], [14].

Authors of [9] have projected the Link based Distribution Multi hop Broadcasting (LDMB), which has same potential forwarders and receivers. Every forwarder calculates and delays for period of contention, and when the period of contention overs then the forwarder would start rebroadcasting of the message, this conception is being used for the further research.

Authors of [11], [12] have proposed forwarding strategy based on position by broadcasting the emergency message, and selecting the best forwarder available. All vehicles which receive that message are potential forwarders. So as to choose the node which will forward the message, every receiver would allocate the contention window (waiting time); the size of window will be the lowest for node which is furthest whereas largest size of window for the node which is nearby i.e., priority for the node which is furthest to be the succeeding forwarder will be given by this protocol.

The last two protocols have the problem, which is each of the receivers of message will calculate the contention window period and will pause for making the rebroadcast though the vehicles which are nearby the sender would do, and making the whole network vehicles active for every received message.

In [13], authors have proposed a protocol known as Emergency Message Dissemination Vehicle (EMDV), by allowing the furthest vehicle inside the range of transmission for making the rebroadcast of message.

Because the position is always changing, choosing a forwarder vehicle is not suitable in VANET also receiving vehicle might turn out of range at time when a message is being sent. That means the message receiver is not able to receive it due to the channel problems such as denial of service or jam, as seen in Fig.3.

Authors of [10] have projected that the message receivers would choose arbitrary waiting periods and will also acknowledge for avoiding retransmission by the nodes which are nearby the initial sender.

The delay for rebroadcasting is being caused by acknowledgment scheme. Authors of [14] have projected the protocol named Contention (waiting time) Based Forwarding (CBF) in which a packet in the form of a broadcasting message is being sent by a vehicle to every neighbour of it. On reception of the packet, forwarding the packet will be contended for by neighbouring vehicle. The node with the highest progress to the target would be having the least waiting period also rebroadcasting of the message would be firstly done by it. If other nodes accept the rebroadcast 
message, they will stop their conflict and will delete the previously received message. This protocol is chiefly offered for forwarding the intervallic safety message (Beacons).

This protocol has an issue that it needs technique for management of waiting time or contention period to every adjacent vehicle, also there is possibility of missing the rebroadcast of a different vehicle by the nearest vehicle to the sender. So that vehicle would be rebroadcasting the message .This is known as hidden node problem. Also this is responsible for broadcasting storm problem.

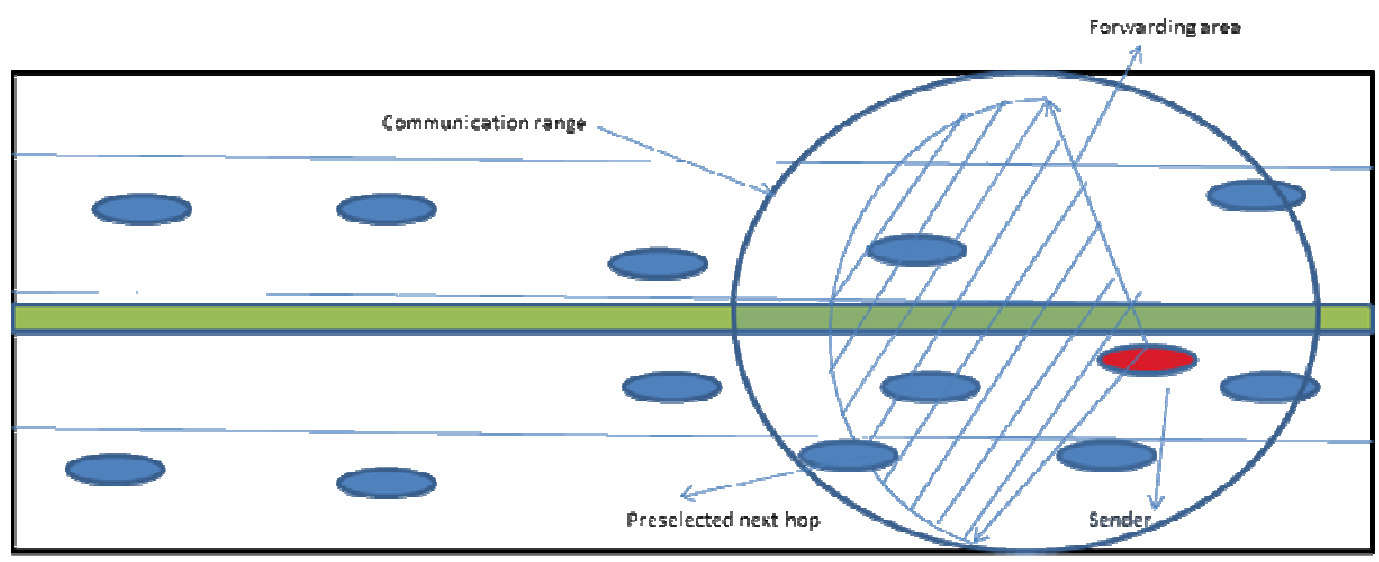

Figure 3.Sender using EMDV

Researchers of [15] have projected that the receivers located at farther distances from the sender would rebroadcast the message by the choice of smaller contention times, as seen in equation.

Authors of [7] have projected a protocol named as Contention(waiting time) Based Broadcasting (CBB) protocol so as to raise the reception of emergency message also to raise the performance, broadcasting is being done in multi-hop manner, prior the sending of original messages, these multi-hop forwarders would be choose. Contention based broadcasting is verified to accomplish supremacy over the as Emergency Message Dissemination Vehicle protocol because it selects many forwarders for rebroadcasting the messages and by this emergency message can defeat the pre chosen forwarder failure.

The standards of picking the forwarders are based on the growth and also based on the localization segment, as seen in fig 4, in which every vehicle positioned in final non-vacant segment become the potential forwarders. 


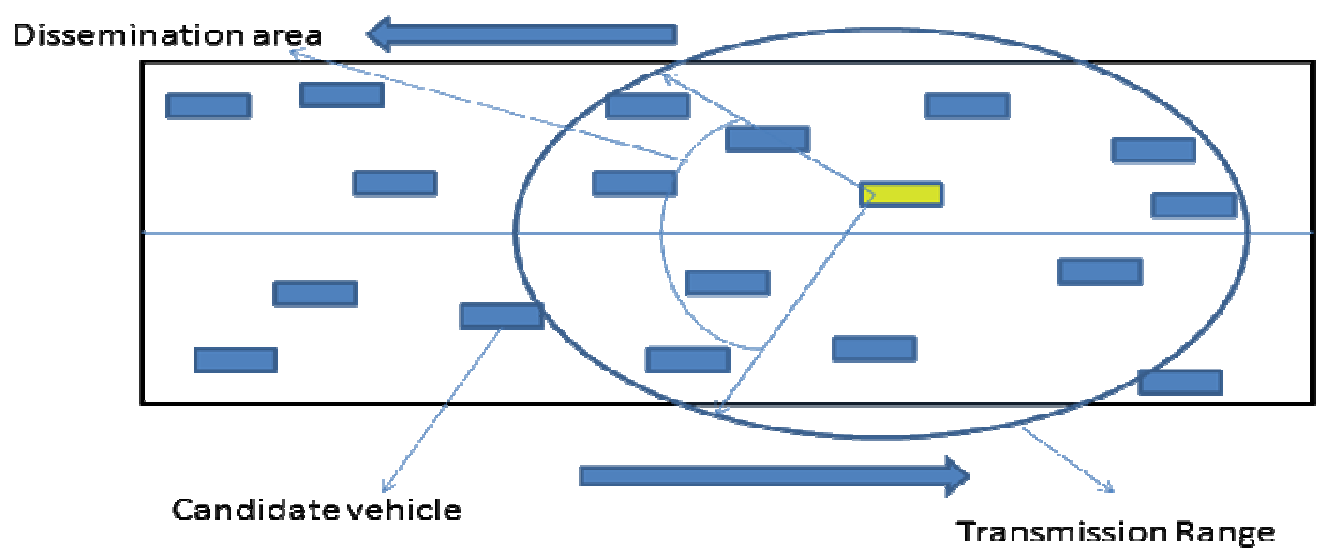

Figure 4. Emergency message Sending and transmission range

Another way to rebroadcast the message is to splits the network into segments proposed in [16, 17, and 18].

The authors of [16] have projected Urban Multi-hop broadcast (UMB) and that increases the message growth, and evading hidden node,problems of broadcasting storm and dependability. It gives the responsibility of furthering and recognising the broadcast packets to only one vehicle. This is being done by separating the road lot inside the communication range into segments and selecting the vehicle in the furthermost non-vacant section without any information of previous topology. Broadcast control packet is being transmitted by source node, named as RTB (Request to Broadcast),and it holds the location of the source and location of the segment size. On reception of the Request To Broadcast packets, the nodes calculate the space amongst the receiver and sender. After that nodes transfers a channel congestion signal known as black burst, which contains numerous time-spaces equivalent to their distance from the source (in number of segments).Farther the distance, lengthier is the black-burst. Each and every node tranfers its congestion signal, when different black-burst is not there in the channel then it results in determining it as the furthest node. A Clear-to-Broadcast (CTB) control packet is returned by node, containing its identifier (ID), to the source.

In [17] authors have projected a protocol which has the similar purpose as that of Urban Multihop Broadcast by making use of a diverse technique. Upon the reception of RTB message, each vehicle should decide its segment and set a random back-off time. Every node is having its unique waiting time.Meaning, if that segment is having the size of contention window of four Time slot,then vehicles which are lying in the farthest segment have to arbitrarily decide a back-off time which lies in between zero time slot and three. The subsequent next segments' vehicles selects the value in between four ansd seven Timeslots. The vehicles which are closer to the sender; they have to wait for longest period of time.

Vehicles will be decreasing their times of backing off by 1 in every Time Slot(Ts). When they are hearing the physical channel. When a Vehicle obtains a suitable Clear To Broadcast packet,when it is listening,the vehicle would be exiting the time phase of contention. And would hear the transmitting broadcast signals. In contrast, suppose a node comes to an end of its back off timer,the node would transfer the Clear-To-Broadcast signal and would be rebroadcasting the incoming broadcasts.

In [20 ]researcher has categorised the message in priority message in which structure of the emergency message is as shown in fig 5 in which 3 inputs, viz. are Forwarder candidate ID (ie., 
Cid), Minimum Boundary and Maximum Boundary, they are supplementary to assist the receiver vehicle in deciding which action should be taken after acceptance of any emergency information or message.

\begin{tabular}{|l|l|l|l|l|l|l|l|}
\hline Send ID & Cd & TS & M ID & Dt & Cnid & MinBt & MaxBt \\
\hline
\end{tabular}

Figure 5.Message Format

Where Send ID : sender id, Cd : Message Code, TS : Time Stamp, MID : Message ID, Dt : Data sent, Cnid : Forwarder Candidate ID, MinBt : Minimum Boundary, MaxBt : Maximum Boundary.

\section{Some Real Time Examples Of Broadcasting Protocols.}

\subsection{Edge-Aware Epidemic Protocol (EAEP):}

Each of vehicles has its unique knowledge which is piggybacked for broadcasting. By this solution, EDGE AWARE EPIDEMIC PROTOCOL operates without beaconing. Upon reception of any recent rebroadcasting information, Edge aware epidemic protocol makes use of number of transmission beginning front nodes and back nodes in amount of time to calculate the probability for making decision whether nodes will rebroadcast the message or not. Using this methodology, the boundary of every communication would be the favoured region for rebroadcasting the message. Although, the intermittent connectivity problem is not being solved by Edge aware epidemic protocol. Particularly, a node will be unknown about if it has overlooked or passed any messages or else its neighbours also have missed some messages it has. EDGE AWARE EPIDEMIC PROTOCOL does better than the simple flooding where as reliability and overhead is concerned. Though, it present time-consuming speed of data dissemination. It needs at least 30 seconds for delivering a broadcast message.

\subsection{Preferred Group Broadcast (PGB)}

PREFRERED GROUP BRODCAST is not a dependable broadcasting protocol. But the protocol is resolution to prevent broadcast storm problem. Every node in PREFRERED GROUP BRODCAST would sense signal strength from broadcasting by neighbour. The signal strength is being used for waiting timeout calculation. Shorter waiting timeout will be set by nodes in the edge of circulated broadcast. Rebroadcasting of the message is being done by the node which is having shortest timeout. PREFRERED GROUP BRODCAST can decrease numbers of RREQ broadcasting. But a problem on low density area still exists.

\subsection{AckPBSM}

AckPBSM is a modified version of PBSM, which is the parameter less broadcast in static to highly mobile ad-hoc networks. This protocol functions by using periodic beacons, which replaces information amongst nodes. In each vehicle cluster, the nodes at either the head or tail would place minimum waiting timeout. The nodes which are lying in Connected Dominating Set are known as gateway nodes. Rest of the nodes which do not lie in Connected Dominating Set would place higher waiting timeout. If all of their neighbours received the messages, the nodes will not perform any rebroadcast. For accurate operation high frequent beacon is needed by this protocol. For solving the problem of intermittent connectivity, broadcasting message 
acknowledgement is being piggybacked in beacons periodically. Therefore, nodes are able to rebroadcast when their neighbours do not get broadcast messages. It is reported in that AckPBSM outperforms PBSM and DVCAST in terms of reliability and overhead..

\subsection{Density-Aware Reliable Broadcasting Protocol (DECA)}

It does not require position knowledge. DECA employs only local density information of 1-hop neighbours obtained by beaconing. Before broadcasting, the node selects one neighbour which has the greatest local density information for being the next rebroadcasting node. And other nodes will randomly set their waiting timeout. If they don't hear anyone rebroadcasting the message before the timeout expiration, they will rebroadcast the message. Also, recognition of the arrived broadcast messages is incorporated into periodic beacons. So as the node can find out its neighbours, who have not yet received the message. The advantage of Density-Aware Reliable Broadcasting Protocol is that, it does not need location knowledge to operate so it is more flexible to suit any operating environment.

\subsection{Position-Aware Reliable Broadcasting Protocol (POCA)}

It uses adaptive beacon to get neighbours' position and velocity. When nodes want to broadcast the messages, they will select neighbours in preferred distance to rebroadcast the message. That chosen distance is selected on the basis of distance among nodes and selector. The selected node will rebroadcast the message instantaneously. In case those selected nodes do not rebroadcast the message, other nodes which are having set waiting timeout since they received message will do this task instead. The waiting timeout is being calculated depending on the distance between node and precursor node. So the node which is closest to selected node will rebroadcast the messages. Position-Aware Reliable Protocol also piggybacks the message attribute to beacon to hold connectivity. Hence a node in the same road section will be rebroadcasting the messages to neighbours.

\subsection{BROADCOMM}

BROADCOMM is projected on the structure that is being utilized in highway network. The highway is being divided into fundamental cells which shift along the vehicles. There is two level of hierarchy for the nodes which are in the highway. All the nodes which are in a cell are included in first level. In each of the virtual cell, some of the nodes signify the subsequent level, which are accountable for managing messages within its cell. And forwarding the communication to or from neighbouring cell reflectors.

- Pros

Outperforms better for simple highway structure which is containing smaller number of nodes.

- Cons

Position information is fully dependent on formation of cells.

\subsection{SECURE RING BROADCASTING (SRB)}

The Secure Ring Broadcasting [5] categorizes the nodes in 3 clusters on the basis of receiving power. That is, Inner Node, Outer node and Secure Ring node. Inner Nodes are those nodes which are nearer to sender. Outer Nodes are the nodes which are farther from sender. Whereas, Secure Ring nodes are those which are at desirable distance from sending node. 
- Pros

To get the more stable routes, minimize number of retransmission messages.

- Cons

Control packet overhead is high.

\subsection{Parameterless Broadcasting In Static Mobile Wireless Ad Hoc(PBSM).}

In PBSM [26], the nodes don't need information of their neighbour. for removing disused broadcasting PBSM uses CDS as well as neighbour exclusion model. The list of neighbouring vehicles which is $\mathrm{R}$ and NR is preserved by every vehicle. Which further assists to identify neighbours which have previously received packet as well as which have not.. When timeout occurs then rebroadcast the packet.

- Pros - It is regardless vehicle position, velocity and direction.

- Cons -Control Packet overhead is high.

\subsection{Distributed Vehicular Broadcast Protocol (DV-CAST).}

Distributed vehicular broadcast protocol is being projected on the connectivity. As merely connected, finely connected and entirely disconnected neighbours which partition vehicles in 3 groups. Whenever region is well connected persistence scheme is used. When region is sparsely connected vehicles are able to instantly rebroadcast along vehicles which are travelling in the same direction subsequent to reception of the broadcast message. When region is totally disconnected vehicles stock up the broadcast message in anticipation of another vehicle come into transmission range. When time is terminated then remove the packets.

- Pros

With using flag variable check whether that packet is redundant or not.

- Cons

- Control overhead is high.

- End to end data transfer delay is also high.

\subsection{Urban Multihop Broadcast Protocol(UMB).}

Urban Multihop Broadcasting protocol [28] is projected to decipher collision and problems of hidden node throughout message transmission in multi hop broadcast. In urban multihop broadcast sender selects the extreme node in broadcast direction so as to forward the packet. It achieves well in higher packet loads \& vehicle traffic density.

- Pros

-Overcomes the Packet collision and hidden node problem.

-Successfully performs at vehicle traffic densities scenarios and higher packet loads.

- Cons

-Waste Bandwidth

\subsection{Vector Based Tracing Detection(V-TRADE).}

V-TRADE is a broadcasting protocol which is based on GPS System, and which categorizes, the neighbours into various groups (forwarding). Just a minute subset of vehicles is chosen for every group for the purpose of rebroadcasting.. 
- Pros

-Bandwidth utilization is improved.

- Cons

-To select the next forwarding node in every hop routing overheads occur.

\subsection{Neighbourhood awareness broadcasting protocols}

In neighbourhood awareness approach, beacon messages are being exchanged to collect necessary information about vehicles in certain neighbourhood (Tonguz et al., 2007; Hao et al., 2008; Sebastian et al., 2010; Peksen and Acarman, 2012. The information or statistics about the vehicle for example position, velocity, ID. Every vehicle would preserve a list of all of its neighbours along entire data which is essential to select next relay computations. Every protocol which uses the above technique is recognised as not being effective where bandwidth is concerned. As general beacon message present a substantial overhead to the network. Follows are examples of such protocols.

\subsection{Media Access Control Protocol (MAC).}

Media Access Control protocol goals in avoiding the extended suspension by letting the furthest away node to be the subsequent relay. Path which lies under the transmission range is distributed in 7 segments. The message is being sent by the sender beside by its location in the header. The positions will be compared by receiver nodes with the position of sender to compute receiver nodes direction with respect to the sender. Merely the nodes which are in the area surrounded by the direction of packet are permitted to partake in the succeeding relay choice. Every contributor node will verify its individual list of neighbour to decide which one lays inside the periphery area. Every contributor node will match its individual segment number with the segments which are in neighbourhood so as to elect whether it is in the segment from which the sender is farthest. The farthest segment from the senders' nodes will be selected as the next relay. If the farthest segment has more than single node, then, each node would be allotted a back off time which is proportionate to the speed of node. However, the elected relay is transmitting the packets; rest of the nodes in the outermost division would carry the message and will work as backup nodes. When transmission comes to the end, backup nodes would verify to match the message or information which is there in its individual buffer which they have acknowledged from the relay. Suppose the message is matched, the nodes would terminate the transmission procedure. Simulation calculates packet response ratio, hop delay and average overhead, for dissimilar node thickness with diverse size of packets. Whereas hop delay and response ratio mark practical values, quantity of the overhead actually rises with traffic. Any other protocol have not been compared with results of other proto cols.

\subsection{Reception Estimation Alarm Routing (REAR) protocol (Hao et al., 2008)}

The probability of reception is used as next reception selection criteria by the protocol, so REAR does not relay the node that provides the largest progress on distance. The receipt probability is maximized which in turn extends the coverage distance and also maintains the quick propagation. By the means of beaconing, neighbours list containing size and position information is maintained by each node. The alarm message including message propagation direction and neighbourhood list of the sender is broadcasted by the sender, only the nodes in propagation direction can participate in the relay node selection, its reception probability is calculated by participant nodes, each node will wait for a contention delay that is inversely proportional to its probability of reception. The node with the shortest contention delay will be elected as a relay and the message is sent further in the network. For more information on the 
International Journal of Grid Computing \& Applications (IJGCA) Vol.6, No.1/2, June 2015

probability of reception calculation refer to Hao et al. (2008). high reliability is shown in Simulation evaluation of this protocol while its performance is affected by its latency delay.

\section{CONCLUSION}

In this paper we have analysis various broadcasting algorithms from various research papers. We can conclude that all broadcasting algorism are considering message transmission as main motive. But while considering this they all are unintentionally neglecting the fact. That is broadcasting improves folding and due to that performance of the system degrades. And one more thing among all this broadcasting protocols no one is providing any security or any technique to reduce accidents happing at the intersection. So our emphasis for future work is to use broadcasting technique for reducing accident at road intersection.

\section{REFERENCES}

[1] Ghassan Samara, R Sures, Wafaa AH Al-Salihy "Security Issues and Challenges of Vehicular Ad Hoc Networks (VANET)", 4th International Conference on New Trends in Information Science and Service Science (NISS), 2010, 393-398.

[2] Ghassan Samara, R Sures, Wafaa AH Al-Salihy "Security Analysis of Vehicular Ad Hoc Nerworks (VANET)", Second International Conference on Network Applications Protocols and Services (NETAPPS), 2010, 55-60.

[3] Who, W. H. (2011), World Health Organization, http://www.who.int/roadsafety/decade_of_action/plan/en/index.html, visited on 30 Apr., 2014.

[4] M Raya, D. J., P Papadimitratos, I Aad, JP Hubaux (2006), Certificate Revocation in Vehicular Network. Laboratory for computer Communications and Applications (LCA) School of Computer and Communication Science, EPFL, Switzerland.

[5] Worldometers (2011), real time world statistics, www.worldometers.info, visited on 30 Apr., 2014.

[6] Ghassan Samara, WAHA Alsalihy, S Ramadass, "Increase Emergency Message Reception in VANET", Journal of Applied Sciences, 2011, Volume 11, Pages 2606-2612.

[7] Ghassan Samara, Sureswaran Ramadass , Wafaa Alsalihy, " Increasing Network Visibility Using Coded Repitition Beacon Piggybacking", World Applied Sciences Journal (WASJ), Volume 13, Number 1, pp. 100 - 108, 2011.

[8] Ching-Yi, Y. \& Shou-Chih, L. (2010) Street Broadcast with Smart Relay for Emergency Messages in VANET. 24th International Conference on Advanced Information Networking and Applications Workshops (WAINA), 323-328, IEEE.

[9] Qiong, Y. \& Lianfeng, S. (2010) A Multi-Hop Broadcast scheme for propagation of emergency messages in VANET. 12th IEEE International Conference on Communication Technology (ICCT), 1072-1075, IEEE.

[10] Biswas, S., Tatchikou, R., Dion, F. (2006), Vehicle-to-vehicle wireless communication protocol for enhancing highway traffic safety, IEEE Communication Magazine, 44, 74-82, IEEE.

[11] Torrent-Moreno, M. (2007), Inter-vehicle communications: assessing information dissemination under safety constraints, 4th Annual Conference on Wireless on Demand Network Systems and Services, WONS '07, 59 - 64, IEEE.

[12] Torrent-Moreno, M., Mittag, P. \& Hartenstein, J., Santi, H. (2009), Vehicle-to-Vehicle Communication: Fair transmit power control for safety-critical information. Transactions on Vehicular Technology, 58, 3684-3703, IEEE

[13] Torrent-Moreno, M. (2007), Inter-Vehicle Communication: Achieving Safety in a Distributed Wireless Environment-Challenges, Systems and Protocols, (Ph.D Paper), Universitatsverlag Karlsruhe, ISBN: 978-3-86644-175-0.

[14] Füßler, H., Kasemann, M., Widmer, J., Mauve, M. \& Hartenstein, H. (2003), Contention-based forwarding for mobile ad hoc networks, Ad Hoc Networks, 1 (2003), 351-369.

[15] Briesemeister, L., Schafers, L., Hommel, G. (2000), Disseminating messages among highly mobile hosts based on inter-vehicle communication, IEEE Intelligent Vehicles Symposium, IV 2000, 522 527, IEEE. 
International Journal of Grid Computing \& Applications (IJGCA) Vol.6, No.1/2, June 2015

[16] Korkmaz, G., Ekici, E., Özgüner, F. \& Özgüner, Ü. (2004), Urban multi-hop broadcast protocol for inter-vehicle communication systems, first ACM international workshop on Vehicular ad hoc network, ACM.

[17] Fasolo, E., Zanella, A., Zorzi, M. (2006), An effective broadcast scheme for alert message propagation in vehicular ad hoc networks, IEEE Int. Conf. on Communications ICC'06,( $3960-$ 3965), IEEE.

[18] Zorzi, M. \& Rao, R. R. (2003), Geographic random forwarding (GeRaF) for ad hoc and sensor networks: energy and latency performance, IEEE transactions on Mobile Computing, (349-365), IEEE.

[19] IEEE (2005), White Paper: DSRC technology and the DSRC Industry consortium (DIC) prototype team.

[20] Intelligent Emergency Message Broadcasting in VANET Using PSO.

\section{AUTHORS}

Mrs Rohini Nere [AhireMe Appear Be computer

Completed her Be From S.S.V.P.S.Coe dhue

Now she is student in Mit Aoe Alandi Pune Doing her me project in Vanet

In Computer Department

S.V.P.S.Coe dhue

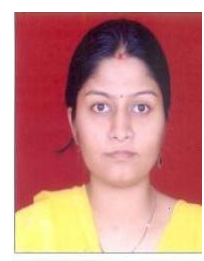

Prof .Mrs Uma Nagaraj

[Phd] Apper Me cmputer

Head of the Computer department in

Mit Aoe Alandi Pune Doing her Phd Research in Vanet

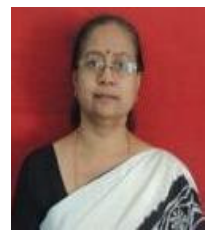

